\title{
The Efficiency of the Moiré Technique for Three-Dimensional Measures of Grape Bunches
}

\author{
Juliana Aparecida Fracarolli ${ }^{1}$, Kátia Cristina Suzigan ${ }^{2}$ and Inácio Maria Dal Fabbro ${ }^{3}$ \\ 1. Department of Postharvest Technology, School of Agricultural Engineering, University of Campinas, Campinas 13083-875, SP, \\ Brazil \\ 2. Department of Pre-processing Agricultural Products, School of Agricultural Engineering, University of Campinas, Campinas \\ 13083-875, SP, Brazil \\ 3. Department of Farm Machinery, School of Agricultural Engineering, University of Campinas, Campinas 13083-875, SP, Brazil
}

\begin{abstract}
This study was carried out in the University of Campinas to study the three-dimensional reconstruction of grape bunches through the application of the Moiré technique as an alternative and innovative method, using low-cost equipment for data acquisition. This study proposed the three-dimensional visualization of grapes and topography on $X_{i}$ axes through the Moiré projection technique. The artificial grapes with a $20 \mathrm{~cm}$ long bunch and $18.45 \mathrm{~mm}$ average diameter for each grape were used. A projector was used to generate a grid with light and dark lines. The grape bunch was placed on a holder, illuminated by a sinusoidal grid and photographed. After processing the images, a three-dimensional map of the fruit and topography on $X_{i}$ axes were obtained. The three-dimensional fruit observation allows the visualization of the higher and lower regions of the three-dimensional configuration through a color map. This result makes it possible to obtain size and shape of the grape bunch, allowing for the process automation of product selection and classification.
\end{abstract}

Key words: Vitis vinifera L., grape bunches, Moiré technique, three-dimensional visualization.

\section{Introduction}

The total surface area planted with vines was 7,534 thousands of hectares, being an important culture [1]. However, grape commercialization is characterized by a short shelf life period. This is due to the fruit characteristics and inadequate postharvest treatment. The fruit usually suffers smashing, threshing and disruption. Inappropriate packaging and transportation methods often cause that kind of mechanical damage. Generally, in Brazil, the grapes are packed in wooden packaging, corrugated cardboard and clamshell packaging, which, in most cases, are in disagreement with the normative instruction in Brazil that defines the fruit packaging characteristics. The most common packaging materials used in grapes transportation are wood and corrugated cardboard [2].

Corresponding author: Juliana Aparecida Fracarolli, assistant professor, research field: postharvest technology.
In grape commercialization, a good visual quality of the bunches is extremely important. Appropriate packaging guarantees quality aspects of the bunches with a lower threshing rate, less weight loss, good bunch and bagasse aspect [3]. For the packaging manufacturing, the fruit three-dimensional reconstruction in a computer is very important.

The three-dimensional modeling allows for the preparation of more adequate packaging for various types of fruit. Moiré is a technique that allows for a three-dimensional visualization of objects.

The phenomenon called Moire is explained by the interference that occurs when light passes through a barrier. The Huygens' principle states that the points of a wavefront behave as point sources for secondary waves.

The purpose of the Moire projection technique studied by Lino [4] was to obtain the Moiré pattern by the superposition of the periodic pattern projection on 
the observation plane with the projected periodic pattern on the object. This technique allowed for the topographic contour restitution of agricultural products with symmetric and asymmetric geometry.

Lino [5] employed a shadow Moiré method to generate a three-dimensional view of a pear fruit surface and compared the results to those obtained with a 3D scanner. However, errors were observed on regions where Moiré fringes were not formed, like on the image background as well as on the object border.

Mazzeti [6] evaluated the deflection of rubber model discs to study sugarcane cutting. When compared the measurements done with the Moiré technique and measurements done with a caliper, a Pearson's correlation coefficient between both techniques of 0.996044 was obtained, indicating good precision.

The reconstitution of a topographic contour with symmetrical or asymmetrical geometry is applied to various fields of sciences, such as biology, orthopedics, human prosthetics and orthotics, medical diagnostics [7], predicting surgical outcomes, veterinary medicine, quality control and industrial inspection, reverse engineering, mathematical modeling, solid mechanics, structural design, machine elements, robotics and machine vision. Moiré techniques have also been developed and applied for deformation measurements on the nanometer scale [8]. In agricultural engineering, several studies try to determine the topographic contour of biological materials from optical techniques, such as

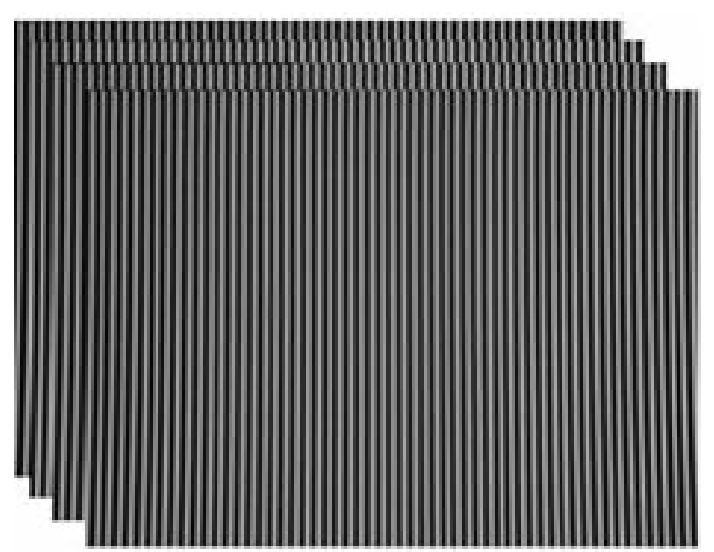

(a)
Moiré [9]; this information can be later used on the classification and selection of agricultural products. Considering the applications of the Moiré technique, the aim of this study was to obtain the three-dimensional reconstruction of a grape bunch through a low-cost technique.

\section{Materials and Methods}

The tests were performed in the optical laboratory of the School of Agricultural Engineering, University of Campinas, SP, Brazil (FEAGRI/UNICAMP) in November 2013. The experiment was carried out on artificial grapes with a $20 \mathrm{~cm}$ long bunch and 18.45 $\mathrm{mm}$ diameter in average for each grape.

On obtaining the Moiré phenomenon, a projector was used to generate a grid with light and dark lines (the dark lines are the obstacles to the light) with a thickness of four pixels, as described by Lino [4]. From this grid, three others were generated with phase difference lines at $1 / 4,1 / 2$, and $3 / 4$ of period compared to the first. Fig. 1a shows the four grids, while Fig. $1 \mathrm{~b}$ illustrates the deformed grid, simulating the projection on a given object.

There are several types of fringes from different types of grids. A very popular grid, called Ronchi grid, consists of parallel and equidistant dark lines interspersed with clear and transparent lines. In this study, sinusoidal grids, $\pi / 2$ radians out-of-phase from each other, were used.

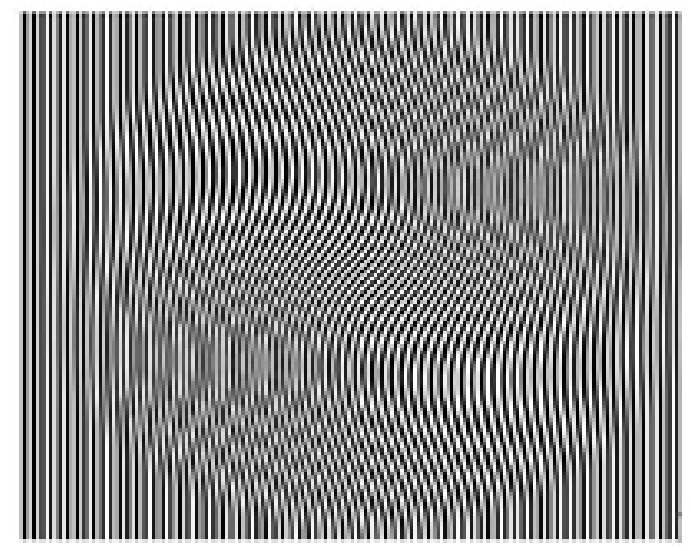

(b)

Fig. 1 Phase grids $1 / 4$ of period different from each other (a) and deformed grid (b).

Source: Lino [4]. 
The Moiré projection technique of grids consists of projecting four sinusoidal grids with phase shifts of 0 , $\pi / 2, \pi$ and $3 \pi / 2$ of period from each other on an object surface. Eqs. (1)-(4) represent successive intensity (I) distributions with phase shifts between each image [10]:

$$
\begin{gathered}
\mathrm{I}_{1}(x, y)=a(x, y)+b(x, y) \cos \varphi(x, y) \\
\mathrm{I}_{2}(x, y)=a(x, y)+b(x, y) \cos [\varphi(x, y)+\pi / 2] \\
\mathrm{I}_{3}(x, y)=a(x, y)+b(x, y) \cos [\varphi(x, y)+\pi] \\
\mathrm{I}_{4}(x, y)=a(x, y)+b(x, y) \cos [\varphi(x, y)+3 \pi / 2]
\end{gathered}
$$

where, $a(x, y)$ is the average intensity, $b(x, y)$ is the modulation of the intensity, and $\varphi(x, y)$ is the phase to be determined.

Solving Eqs. (1)-(4), the phase $\varphi(x, y)$ at each point $(x, y)$ in the frame can be obtained by the following Eq. $(5)$ :

$$
\varphi(x, y)=\arctan \frac{\mathrm{I}_{4}(x, y)-\mathrm{I}_{2}(x, y)}{\mathrm{I}_{1}(x, y)-\mathrm{I}_{3}(x, y)}
$$

The phase obtained in Eq. (5) has a value between $-\pi$ and $\pi$. The phase demodulation is performed in order to remove phase ambiguities. This is done by adding or subtracting a value of $2 \pi$ to the individual pixel, until the phase difference between the adjacent pixels is less than $\pi[11]$.

In this study, an artificial grape bunch was used. The Moiré technique was applied to a body with complex geometry with concave regions, like a grape bunch. The object of study was painted flat white in order to provide greater contrast between the Moiré fringes and to make image acquisition easier. For image acquisition, as shown in Fig. 2, the experimental apparatus we developed with the following components: (1) white light source (wavelength between 400-700 nm), multimedia projector with a 170 $\mathrm{W}$ power lamp; (2) digital camera (Samsung SC-HMX20C with a 6.4 megapixels sensor); reference plane.

The Moiré projection technique is the fringe projection on the reference plane and the object of study. The projected fringes were generated by the public domain Fringe Projection software. The sinusoidal fringe projection was opted in order to avoid some errors found in rectangular fringes.

Four color images of the plan with the Moiré fringe were obtained. Between each image taken, the sinusoidal fringe was shifted from 0 to $\pi / 2, \pi$ and $3 \pi / 2$ (images $\mathrm{I}_{1}, \mathrm{I}_{2}, \mathrm{I}_{3}$ and $\mathrm{I}_{4}$ ). After fixing the object of study on the reference plane, just one image with the sinusoidal fringe projection without displacement $\left(\mathrm{U}_{1}\right)$ was taken. Fig. 3 shows the images.

In the image processing, the software Image J v1.47u [12] and Moiré Analysis Software v0.87 (MAS), both public domain, were used. The ImageJ software was used to run program subroutines, perform arithmetic operations and display the treated image in three dimensions. The MAS software was used in the four images juxtaposition of phases shift with the phase-shift filtering tool.

The images went through the following process:

(1) Image conversion to grayscale, with 256 grayscales, ranging from 0 (black) to 255 (white).

(2) Calculation of subtraction module to duplicate

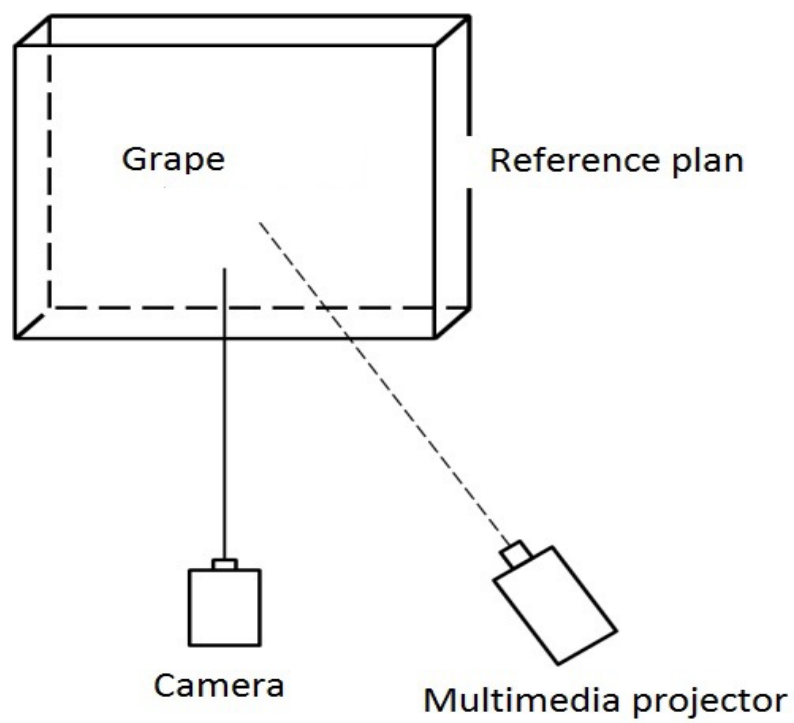

Fig. 2 Experimental setup for the Moiré projection technique implementation. 

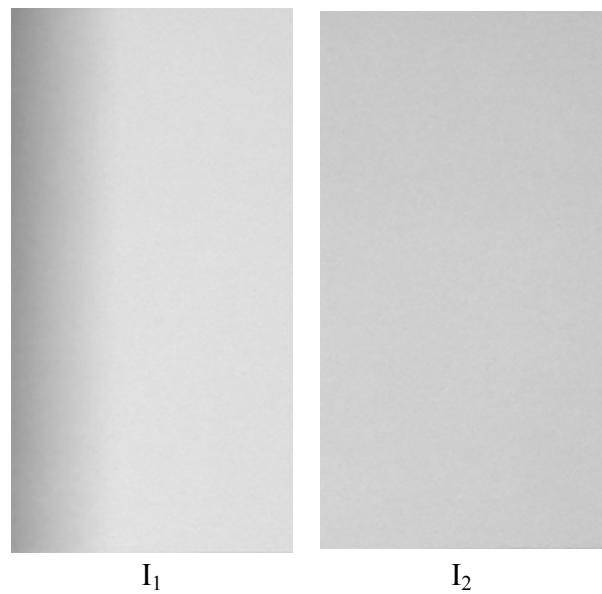

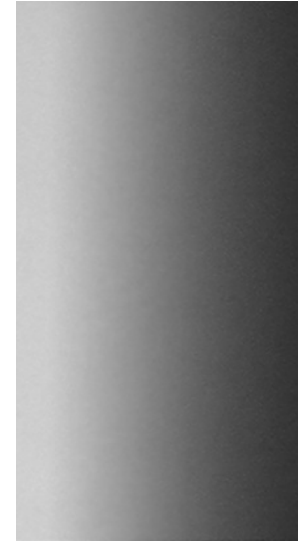

$\mathrm{I}_{3}$

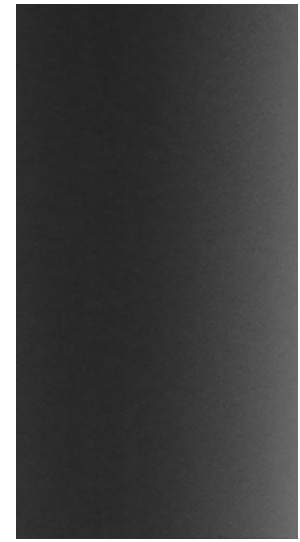

$\mathrm{I}_{4}$

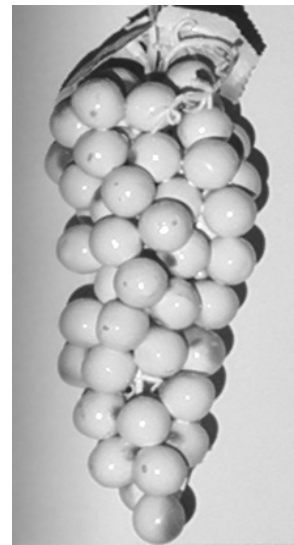

$\mathrm{U}_{1}$

Fig. 3 Images of sinusoidal fringe in the offset plane of $0\left(I_{1}\right), \pi / 2\left(I_{2}\right), \pi\left(I_{3}\right)$ and $3 \pi / 2\left(I_{4}\right)$, and image of the grape bunch $\left(U_{1}\right)$.

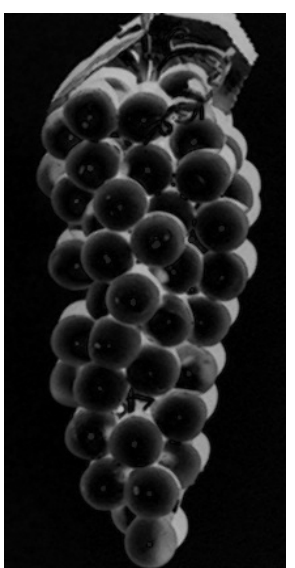

(a)

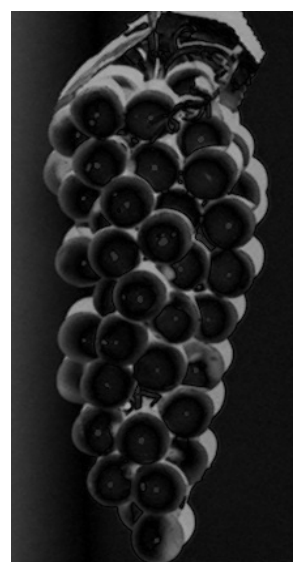

(b)

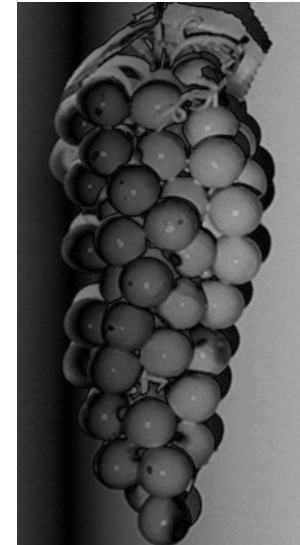

(c)

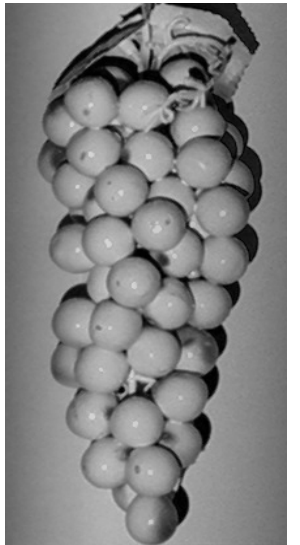

(d)

Fig. 4 Images with phase shift of $0(a), \pi / 2(b), \pi(c)$ and $3 \pi / 2$ (d).

and highlight the fringes on the object surface between the images of the plane and the object. The difference between each plane image $\left(\mathrm{I}_{1}, \mathrm{I}_{2}, \mathrm{I}_{3}\right.$ and $\left.\mathrm{I}_{4}\right)$ was performed, in relation to the object image $\left(U_{1}\right)$. Fig. 4 shows the result of this procedure.

(3) Superposition of images with fringes (MAS > displacement $>$ calculate displacement $>$ calculate displacement from phase) for relief image (Fig. 5a). At this point, Eqs. (1)-(5) are used.

(4) Image unwrapping to remove the discontinuities (Fig. 5b).

(5) Three-dimensional map with the function interactive 3D surface plots in ImageJ software.

\section{Results and Discussion}

The grape berries were visualized in Fig. 6. The color close to the blue hue represents points of more portions in $X_{3}$, and the color close to the red hue represents the points of minor portion in the $X_{3}$ axis.

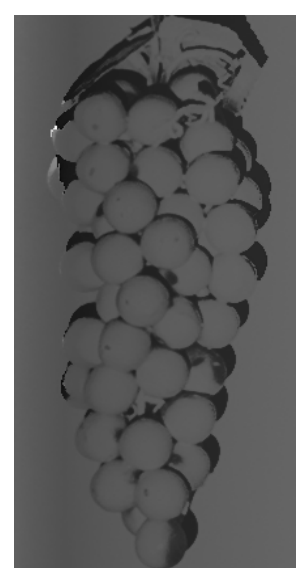

(a)

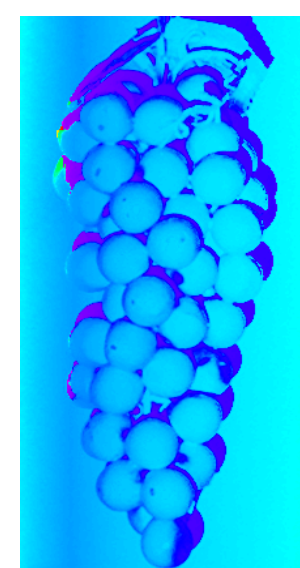

(b)
Fig. 5 Map of phases and their discontinuities (a) and the retrieved surface of the unwrapping result (b). 


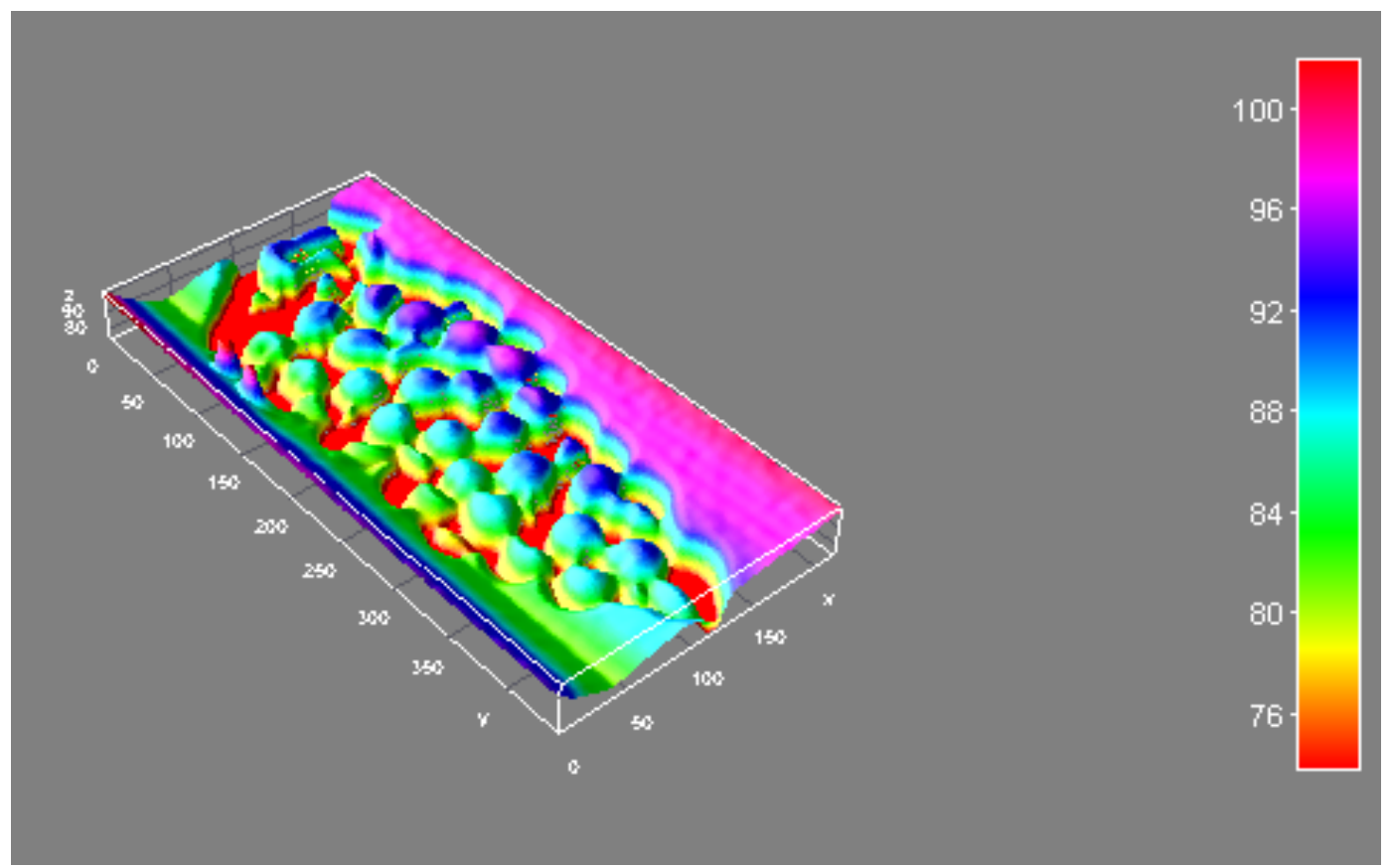

Fig. 6 Three-dimensional visualization with topography information of the object.

The color bar indicates the deep in $X_{3}$ axis.

A $20 \mathrm{~cm}$ long grape bunch with an average diameter of $18.45 \mathrm{~mm}$ for each grape was used as testing body in this work, in which the $3 \mathrm{D}$ complexity generates shadow regions in the empty spaces where Moiré fringes are not generated. The results show noises at those shadow areas, like between grapes and the bunch contour. The grape top areas showed better 3D reconstruction. The three-dimensional fruit observation allows for the visualization of the higher and lower regions of the three-dimensional configuration through a color map. Further studies should be done in dark colored fruit in order to determine the efficiency of the technique on fresh fruit.

The 3D model obtained through the Moiré technique can generate a mathematical description of the grape bunch. From the three-dimensional computer reconstruction of the fruit, it is possible to develop better packages. Thus, it is also possible to accomplish improvements on transport, reducing mechanical damages to the fruit and postharvest.

This analysis is qualified as a non-destructive method, which does not require physical contact of the object with any instruments. So, it is also possible to do the fruit classification and selection.

Lino [4] reported the application of Moiré techniques in surveying the topography of spherical shells, with good agreement to real values. The author emphasizes that Moiré methods are sensitive enough to recognize peaks and valleys on the surface under study.

Costa et al. [13] used the Moiré technique to estimate the volume of macaw palm fruit and compared the Moiré technique to the displacement method of a fluid in order to obtain the volume of the fruit. Lino and Fabbro [14] applied the Moiré technique to obtain the topography of artificial fruit made of paraffin on a 1:1 scale. The authors obtained a digital model of pears through the Moiré technique and compared it to the three-dimensional model obtained through a laser scanner. In both cases [13, 14], the authors found a good correlation between the three-dimensional model obtained through the Moiré technique and the comparison methods, which were volume displacement of a fluid [13] and the 3D model obtained through a laser scanner [14]. 


\section{Conclusions}

The Moiré projection technique allowed us to generate a grape bunch visualization and topography on $X_{i}$ axes. The topography on $X_{i}$ axes made it possible to obtain the size and shape of the grape bunch. This technique allowed for the three-dimensional reconstruction through low-cost equipment and the minimal computational processing.

\section{References}

[1] International Organization of Vine and Wine (OIV). 2016. "State of the Vitiviniculture World Market." Accessed April, 2016. http://www.oiv.int/public/medias/4710/oivnoteconjmars2016-en.pdf.

[2] Gomes, D. 2006. "The Effects of Vibration in the Quality of Niagara 'Rosada' Grape." Master thesis, University of Campinas. (in Portuguese)

[3] De Castro, J. V., Park, K. J., and Honório, S. L. 1999. "Use of Packaging for Postharvest Conservation of Grapes." Rev. Bras. Eng. Agric. Ambient. 3 (1): 35-40. (in Portuguese)

[4] Lino, A. C. L. 2008. "Applications of Projection Moire Technique with Phase Shifiting to Agricultural Engineering Problems." Doctoral thesis, University of Campinas. (in Portuguese)

[5] Lino, A. C. L. 2002. "Utilization of Moiré Techniques for Fruit Topographic Determination." Master thesis,
University of Campinas. (in Portuguese)

[6] Mazzeti, F. V. 2004. "Use of Moiré Interferometry in the Study of Dynamic Stresses on Floppy Disks." Master thesis, University of Campinas. (in Portuguese)

[7] Porto, F., Gurgel, J. L., Russomano, T., and Farinatti, P. D. T. V. "Moiré Topography: Characteristics and Clinical Application." Gait and Posture 32 (3): 422-4.

[8] Liu, Z. W., Xie, H. M., Fang, D. N., Shang, H. X., and Dai, F. L. 2004. "A Novel Nano-Moiré Method with Scanning Tunneling Microscope (STM)." J. Mater. Process. Technol. 148 (1): 77-82.

[9] Costa, R. M., Braga, R. A., Oliveira, B. S., Silva, E., Yanagi, T., and Lima, J. T. 2008. "Sensitivity of the Moiré Technique for Measuring Biological Surfaces." Biosyst. Eng. 100 (3): 321-8.

[10] Creath, K. 1988. "V Phase-Measurement Interferometry Techniques.” Prog. Opt. 26: 349-93.

[11] Miao, H., Quan, C., Tay, C. J., and Fu, Y. 2007. "Analysis of Phase Distortion in Phase-Shifted Fringe Projection." Opt. Lasers Eng. 45 (2): 318-25.

[12] Schneider, C. A., Rasband, W. S., and Eliceiri, K. W. 2012. "NIH Image to ImageJ: 25 Years of Image Analysis." Nat. Methods 9: 671-5.

[13] Costa, A. G., Ribeiro, E., Braga, R. A., and Pinto, F. A. C. 2016. "Measurement of Volume of Macaw Palm Fruit Using Traditional and the Digital Moiré Techniques." Rev. Bras. Eng. Agric. Ambient. 20 (2): 152-7.

[14] Lino, A. C. L., and Fabbro, I. M. D. 2004. "Fruit Profilometry based on Shadow Moiré Techniques." Ciênc. Agrotec. 28 (1): 119-25. (in Portuguese) 